\title{
The differentiation of peripheral effector neuron failure from acute brain stem dysfunction in a critically ill patient
}

\author{
P G Bain, G Harwood, G E Holder, B M Evans
}

\begin{abstract}
A patient appeared to be in coma following pneumococcal meningitis, an intracerebal haemorrhage, and a cardiac arrest. Late in the course of his illness neurophysiological investigations confirmed a proposal that he also had a fulminant acute demyelinating polyneuropathy which, for a period of five days, rendered the patient completely unresponsive. The patient recovered and is now working without any disability.
\end{abstract}

\section{Case history}

A fit 38 year old man slipped and fell about two metres, landing on his back. He immediately experienced some lumbar pain but not sufficient to prevent him continuing his day's work. That evening his general practitioner prescribed analgesics and bed rest. The symptoms remained unaltered for three days but then, over a period of 72 hours, he became increasingly confused and developed rigors, vomiting, generalised myalgia and neck stiffness.

Six days after his accident he was admitted to hospital where drowsiness, a pyrexia of $39^{\circ} \mathrm{C}$ and a tachycardia of 120 beats/minute were noted. There was also marked neck stiffness, photophobia, tenderness over the lumbar spine and reduced straight leg raising (to $30^{\circ}$ bilaterally), but no focal neurological signs. Initial investigations revealed: ESR

South-East Thames Regional

Neurosciences Centre,

The Brook Hospital,

London, UK

Department of

Neurology

P G Bain

G Harwood

Department of

Clinical

Neurophysiology

G E Holder

B M Evans

Correspondence to:

Dr Bain, MRC Human

Movement and Balance Unit,

The Institute of Neurology,

Queen Square, London

WCIN 3BG, UK

Received 12 April 1991

and in revised form

11 October 1991.

Accepted 28 October 1991 and intravenous chloramphenicol (1 gram, six hourly for three days) and benzylpenicillin (2 grams four hourly for seven days) administered. He became apyrexial but when, one week after the instigation of therapy, he developed a left ptosis, a cerebral abscess was suspected and he was transferred to the regional neurological unit.

On arrival he was drowsy and sweating profusely. He complained of intense back pain and photophobia in an incoherent mumble. Examination revealed a temperature of $37^{\circ} \mathrm{C}$, a pulse of 130 beats/minute and a blood pressure of $180 / 90 \mathrm{mmHg}$. The presence of bilateral papilloedema, neck stiffness, Kernig's sign, tenderness over the whole spine and diplopia on extreme left and right gaze were noted. He was able to flex all four limbs to pain, albeit sluggishly, and had flexor plantar responses with hypertonia and hyperreflexia in the left-sided limbs. Further radiographs of his skull, whole spine and chest confirmed the previous findings whilst an ECG showed Möbitz type one $2^{\circ}$ heart block. Within 30 minutes of his arrival on the unit he had a cardiorespiratory arrest, became asystolic, aspirated and collapsed his right lung. The resuscitation lasted over twenty minutes, during which he was cyanosed and acidotic (arterial blood gases: $\mathrm{po}_{2} 5 \mathrm{Kpa}, \mathrm{pco}_{2} 15 \mathrm{Kpa}$ and $\mathrm{pH} 7 \cdot 0$ ).

He was transferred to the intensive care unit (having been intubated during the resuscitation) where, under a general anaesthetic, a bronchoscopy was performed and a thick plug of vomit removed from the right main bronchus. This resulted in the re-expansion of the right lung and improved arterial blood gases $\left(\mathrm{po}_{2} 7.9 \mathrm{Kpa}, \mathrm{pco}_{2} 7 \cdot 47\right.$ and $\mathrm{pH} 7 \cdot 33$ ). Intravenous dexamethasone and broad spectrum antibiotics were introduced and the patient was ventilated electively for the next 24 hours. A cerebral CT scan showed a right frontal intracerebral haematoma with some midline displacement to the left (fig 1). It was not possible to wean him off the ventilator. Despite the possiblity of CNS damage resulting from the cardio-respiratory arrest, frontal haematoma and the referring hospital's diagnosis of pneumococcal meningitis a central explanation for his apnoea was considered to be untenable because he was able to answer simple questions by shutting his eyes once for 
Figure 1 A cerebral CT scan showing a right frontal intracerebral haemotoma with some displacement of the midline to the opposite side.

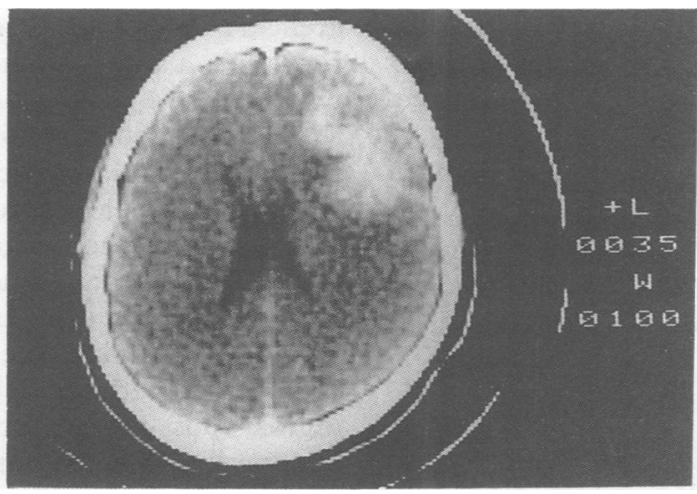

"yes" and twice for "no". Moreover, using this method, the patient was able to indicate that he was unable to move his limbs. Bilateral papilloedema was present and tendon reflexes were now absent. Over the next five days he became progressively less responsive.

Re-examination revealed fixed dilated pupils, bilateral papilloedema, absent eye movements (including caloric responses), global areflexia and total immobility. Over the next five days repeated examinations revealed identical results and on one occasion an anaesthetist found no respiratory movements (final postdisconnection $\mathrm{pco}_{2} 8.5 \mathrm{Kpa}$ ) on testing for apnoea (in the approved manner ${ }^{1}$ ). This test was not repeated as is required for certifying brain stem death under the current UK criteria. An anaesthetist's "peripheral nerve stimulator" produced normal responses from the abductor pollicis brevis muscles on stimulating the median nerves at the wrists. There was no question of sedative drugs or muscle relaxants complicating the clinical picture. This period was complicated by gross instability of his pulse and blood pressure.

An EEG showed a normal alpha rhythm which blocked on arousal. Focal delta activity was present over the right frontal region. Brain stem auditory evoked potentials (BSAEPs) from the right ear were normal whilst those from the left showed a probable mild wave $V$ abnormality (fig 2) suggesting that the brain stem was functioning adequately. Stimulation of the peripheral nerves six days after the cardiac arrest failed to elicit motor responses from right abductor policis brevis (RAPB), abductor digiti minimi, peroneal or facial nerves. EMG studies provided positive evidence of denervation: frequent positive sharp waves and fibrillation potentials were present in the right tibialis anterior, extensor digitorum communis and first dorsal interosseous muscles, and similar findings were present on day 14 post arrest. The severity of the autonomic involvement was illustrated by a $60 \mathrm{mmHg}$ rise in his diastolic blood pressure when click stimulation was applied for the recordings of his BSAEPs.

During the next three weeks the patient improved and was weaned off the ventilator. This improvement was mirrored by the results of further nerve conduction studies. On day 27 motor responses were obtained from RAPB stimulating the median nerve at the wrist and

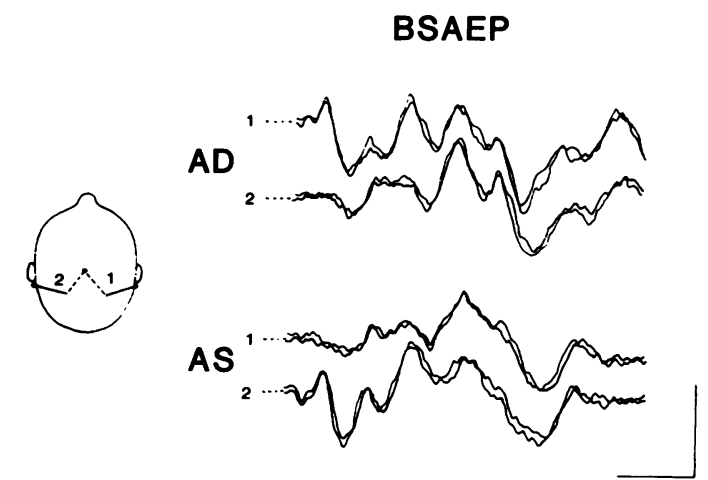

Figure 2 Brain stem auditory evoked potentials (70dB nHL rarefaction phase clicks; 20.3 per second): $A D$ (from the right ear) are normal, $A S$ (from the left ear) shows a probable mild wave $V$ abnormality.

elbow (amplitudes $120 \mu \mathrm{v}$ and $100 \mu \mathrm{v}$, conduction velocity $9 \mathrm{~m} / \mathrm{s}$ ). The conduction velocity was $30 \mathrm{~m} / \mathrm{s}$ on day 58 , when the patient was fully ambulant. The sensory action potentials from median and ulnar nerves were absent throughout. These findings were in keeping with a resolving demyelinating polyneuropathy. He continued to regain his strength and four months later returned home to his wife and then work. Nine months after his arrest the only residual symptom was painful paraesthesiae in his feet. The ankle reflexes remained absent.

\section{Discussion}

Even with hindsight it is difficult to discover exactly what happened to our patient. A reasonable hypothesis is that a subclinical neuropathy caused him to slip and fall. As the neuropathy worsened it was complicated by respiratory failure and autonomic lability which in turn resulted in an intracerebral haemorrhage. This synopsis avoids making the diagnosis of meningitis which was, in the circumstances, quite understandable. The patient had signs of meningism, a source of infection (pneumonia) and an abnormal CSF, with a high percentage of polymorphs. An acute idiopathic demyelinating polyneuropathy could account for the elevated CSF protein concentration ( 1.6 grams $/ \mathrm{L})$, his severe back pain, gross autonomic instability and cardiac arrest, as these are documented features of this disease. It does not, however, explain the elevated CSF cell counts, which with meningism and papilloedema are more easily explained by the combined effects of his intracerebral haemorrhage, Guillain-Barré syndrome (GBS) and septicaemia.

The patient was fortunate that he had a period after his cardiac arrest, during which we appreciated that his conscious level was disproportionate to the severity of his motor signs. (He was able to blink in response to simple questions/commands). This "lucid" interval and the alteration in his physical signs, from left sided hypertonia and hypereflexia to a flaccid quadreparesis, prompted the diagnosis of an acute polyneuropathy.

Once the patient had become completely 
unresponsive we were unable to determine with clinical examination techniques whether his unresponsiveness was due to brainstem dysfunction or effector neuron failure. We were also unable to make any clinical assessment of CNS function. The brain stem death tests ${ }^{12}$ all depend on an intact "final common pathway", and are inappropriate if this pathway is damaged. They are incapable of assessing the afferent neuronal pathways independently. This can be achieved by the use of brain stem auditory evoked potentials or short latency somatosensory potentials. ${ }^{3}$ Our findings of virtually normal BSAEPs and in particular normal I-III interpeak intervals is perhaps surprising as the myelin of the auditory component of the eighth cranial nerve is derived from oligodendrocytes proximally and Schwann cells distally. ${ }^{4}$ The general assumption that the nerve will be spared in every patient with GBS is dubious as there is conflicting evidence on this point. ${ }^{5-9}$ Nevertheless if waves I and II of the BSAEP are present assessment of brain stem function is possible despite a defective motor system.

A curious anomaly in our case was the anaesthetist's finding of "normal" responses from the abductor pollicis brevis muscles when the median nerves were stimulated at the wrists, with a "twitcher", whereas twenty four hours later no responses could be obtained during formal nerve conduction studies. Perhaps the "twitcher" stimulated the thenar muscles directly, alternatively this discrepancy may have reflected the development of conduction block.

The relevance of this case to the current UK criteria for establishing brain stem death ${ }^{1210}$ is to suggest that these sound, pragmatic guidelines need to be applied judiciously. In particular the use of neurophysiological techniques should be considered if a patient is areflexic and flaccid as these signs could represent a lower motor neuron lesion from which a good recovery is potentially possible. We suggest that areflexia and flaccidity should render the brain stem death tests inappropriate without further neurophysiological assessment. This precondition should apply irrespective of whether or not a cerebral or brainstem lesion is present. Indeed, here was a man who had three plausible intracranial causes for brain stem death, namely pneumococcal meningitis, post-arrest cerebral anoxia and an intracerebral haematoma all of which proved peripheral to the main problem which was an unusually severe acute demyelinating polyneuropathy. The vital clue was the disparity, albeit temporary, that existed between his conscious level and the other physical signs.

We thank Professor R A C Hughes for his advice and Elizabeth Chitty for her help in typing the manuscript.

1 Pallis C. ABC of brain stem death: Diagnosis of brain stem death II. BMJ 1982;285:1593-676.

2 Pallis C. ABC of brain stem death: Diagnosis of brain stem death I. BMJ 1982;285:1558-60.

3 Facco E, Casartelli Liviero $M$, Munarri $M$, Toffoletto $F$, Baratto F, Giron GP. Short latency evoked potentials: new criteria for brain death? J Neurol Neurosurg Psychiatry 1990;53:351-3.

4 Kadlubowski M, Hughes RAC, Gregson NA. Spontaneous and experimental neuritis and the distribution of the and experimental neuritis and the distribution of the myelin protein

5 Ropper AH, Chiappa KH. Evoked potentials in GuillainBarré syndrome. Neurol 1986;36:57-590.

6 Schiff JA, Cracco RQ, Cracco JB. Brainstem auditory evoked potentials in Guillain-Barré syndrome. Neurol 1985 35:771-3.

7 Carroll WM, Mastaglia FL. "Locked-in coma" in postinfective polyneuropathy. Arch Neurol 1979;36:46-7.

8 Drury I, Westmoreland BF, Sharbrough FW. Fulminan demyelinating polyradiculoneuropathy resembling brain death. Electroencephalography and Neurophysiology 1987 67:42-3.

9 Kotsoris H, Schleifer L, Menken M, Plum F. Total Lockedin state resembling brain death in polyneuropathy. Annals in state resembling brain

10 O'Brien MD. Criteria for diagnosing brain stem death. BMJ 1990;301:108-9. 